\title{
AVALIAÇÃO PÓS-OCUPAÇÃO (APO) NO BRASIL, 30 ANOS: O QUE HÁ DE NOVO?
}

POST=OCCUPANCY EVALUATION (POE) IN BRAZIL, 30 YEARS: WHAT'S NEW?

\section{ORNSTEIN, SHEILA WALBE}

Professora titular da Faculdade de Arquitetura e Urbanismo da Universidade de São Paulo,

bolsista do Conselho Nacional de Desenvolvimento Científico e Tecnológico (CNPq), email: sheilawo@usp.br

\section{RESUMO}

Este ensaio faz uma breve reflexão crítica sobre o estágio atual das pesquisas em Avaliação Pós-Ocupação (APO) no Brasil e abre a discussão sobre as lacunas acadêmicas ainda a serem preenchidas.

\section{ABSTRACT}

This essay deals with a critique about the today phase of the Post-Occupancy Evaluation (POE) researches in Brazil and propose a discussion about the academic gaps on the field still to be fulfilled. 


\section{TRINTA ANOS: POUCAS TEORIAS, AVANÇOS NAS ABORDAGENS METODOLÓGICAS}

Avaliar, como procedimento de gestão e monitoramento da evolução e da qualidade de um dado programa (por exemplo, social, educacional e assim por diante), de um dado produto (por exemplo, um veículo a ser colocado no mercado) e ainda de indivíduos (por exemplo, estudantes, quanto a sua evolução pedagógica), não é uma novidade.

Há décadas, como parte da gestão pública e privada, os setores que mais prezam a qualidade e a segurança dos usuários que terão contato com os programas ou produtos por eles promovidos e colocados à venda/distribuídos, passam, necessariamente, por avaliações dos seus processos e dos seus produtos, notadamente nos países desenvolvidos. Nestas avaliações, o ponto de vista e a satisfação dos usuários associados ao monitoramento e aferição das metas a serem alcançadas, integram os protocolos investigativos. Nestes casos, o tratamento estatístico dos dados, tem se mostrado fundamental, com vistas a correções, ajustes e aperfeiçoamento dos processos e dos produtos (ROSSI; FREEMAN, 1985). Aqui, as análises da relação custos-benefícios são levadas em conta, bem como o efetivo alcance das metas propostas em função da população alvo (os usuários).

No caso de ambientes construídos e em uso, a APO tem sido aplicada na Europa e Estados Unidos desde o Pós II Guerra, período em que foi verificada a necessidade dos ambientes concebidos e construídos, além de atenderem ao arcabouço normativo e legislativo vigente, satisfazerem às expectativas dos usuários com vistas ao alcance da habitabilidade, do conforto e do bem-estar (ORNSTEIN, 1996).

No Brasil, a APO, enquanto pesquisa aplicada e voltada para valorização da relação "especialistas versus usuários" teve início nos anos 1980 e tem percorrido um longo processo de consolidação e amadurecimento em termos de ensino e pesquisa, sobretudo nas universidades públicas. De modo geral ela tem se mostrado uma contribuição bastante significativa ao campo da Tecnologia da Arquitetura e do Urbanismo (TAU), no qual as "áreas" para pesquisa (ainda) são relativamente poucas, à exceção da computação gráfica, da modelagem 3D e do Building Information Modeling (BIM).

Talvez seja possível afirmar que a APO, nos últimos anos, contribuiu em particular para uma ruptura acadêmica do paradigma sobre o processo de produção, uso, operação e manutenção de ambientes construídos, até então considerados sob uma ótica convencional e linear, de etapas sequencias (Figura 1) e a substituição desse paradigma por uma visão realimentadora em círculos ou mesmo em espiral, em prol das melhorias continuadas dos ambientes construídos (Figura 2).

Assim, em que pese a consolidação e a disseminação da APO no meio acadêmico, a complexidade de avaliar os ambientes construídos que compõem a cidade (um edifício, uma praça ou uma quadra urbana, dentre outros) não tem sido suficiente para promover significativos avanços teórico-conceituais e de abordagem metodológicas capazes de imprimir, entre seus pesquisadores, disseminação semelhante àquela ocorrida nos anos 1980 e 1990. Assim, atualmente ainda permanecem diversos "gaps" a serem preenchidos, inclusive para que a APO, enquanto conjunto de procedimentos inerentes à gestão da qualidade no processo de projeto, uso, operação e manutenção de ambientes construídos, seja efetivamente absorvida na prática profissional e pelos gestores de empreendimentos ou facilities (públicos ou privados).

Figura 1: Visão Convencional e Linear do Processo de Projeto, Construção, Ocupação, Uso, Operação e Manutenção do Ambiente Construído.

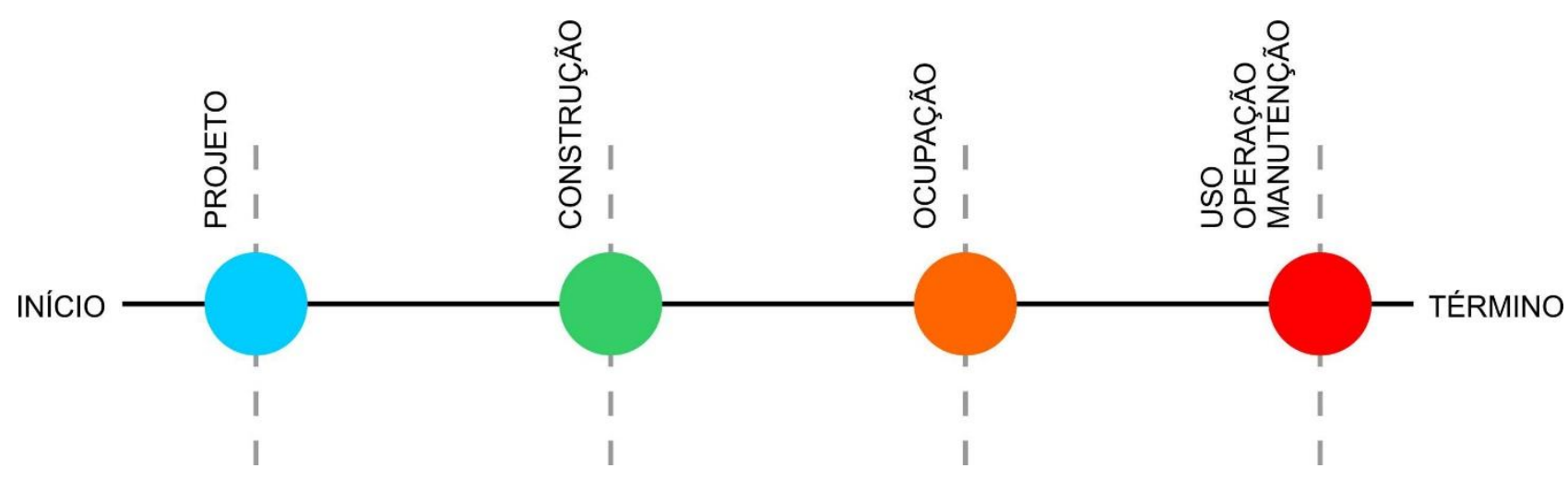

Fonte: A autora. 
Figura 2: Visão Contemporânea do Processo de Projeto, Construção, Ocupação, Uso, Operação e Manutenção para a Melhoria Continuada do Ambiente Construído.

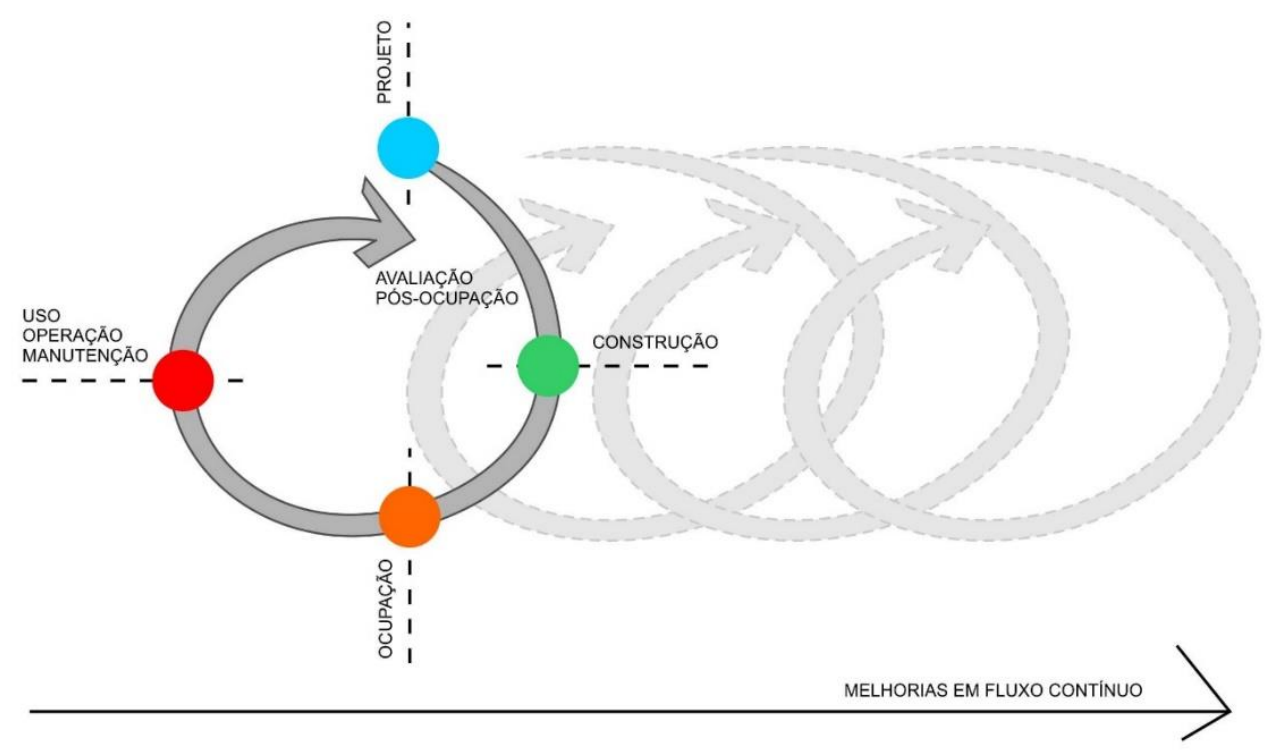

Fonte: A autora.

Por outro lado, na medida em que, de per si, a complexidade dos ambientes construídos (desde a unidade habitacional unifamiliar até aqueles altamente especializados e que atendem a diversas categorias de usuários, como hospitais e museus) exige sua permanente manutenção e adequação às expectativas de seus usuários, as pesquisas de APO têm assumido cada vez mais o seu caráter interdisciplinar (ZEISEL, 2006). Além disso, as dinâmicas e mutações sócio-culturais de cada lugar ou locus (RHEINGANTZ, ROSA, SZAPIRO, 2016) igualmente exigem a aplicação da APO de modo academicamente correto, mas sem os vieses de receituários únicos, o que torna essencial o desenvolvimento de um olhar treinado do pesquisador para perceber o "outro", usuário do ambiente, sem refletir, de forma contaminante, a sua própria trajetória na pesquisa.

Neste contexto, muitos pesquisadores dedicados a APO demonstram de forma recorrente que, mesmo com a ampla disseminação de pesquisas, dissertações, teses, livros (inclusive pela Internet) sobre 0 assunto, nos âmbitos nacional e internacional, há, ainda, uma grande reprodução de estudos de casos e seus resultados, tais como eram desenvolvidos há 20 ou mais anos atrás, situação especialmente evidente nas avaliações de habitações de interesse social e de edifícios escolares.

Afinal, chegou-se a uma encruzilhada?

\section{APROXIMAÇÕES ENTRE ABORDAGENS METODOLÓGICAS DA APO E AS PESQUISAS EM DESIGN}

Certamente não se trata de uma encruzilhada, mas de um "ponto de inflexão" para a APO, em que os avanços nas pesquisas irão ocorrer de modo mais lento e com foco nos incrementos metodológicos, sobretudo dos instrumentos e das ferramentas. Nesta direção, vale a pena lembrar e tratar como uma oportunidade, a aproximação entre os pesquisadores nos campos do design (sob a ótica da ergonomia) e da arquitetura (sob a ótica da APO).

Lawson (2011) em sua obra, já aborda a possibilidade de arquitetos e designers enfrentarem os problemas nas suas respectivas práticas profissionais de forma semelhante. Norman (2006) destaca o projeto centrado no usuário e as diferenças daquele centrado no objeto ou produto. Dijon (2010), destaca a complexidade do campo do design e a necessidade de se considerar, para 0 desenvolvimento de conceitos de produtos, as diversas dimensões em que o mesmo está inserido, como a ambiência, a cultural, a social, o mercado e assim por diante, tal como acontece nos projetos de arquitetura e de urbanismo. Abrahão et al. (2009) destacam o método da Análise Ergonômica do Trabalho (AET) e Mont Alvão e Villarouco propõem a incorporação da AET à Avaliação Ergonômica do Ambiente Construído (AEAC), aproximando de forma consistente os procedimentos metodológicos da APO (com origem na Arquitetura) da AEAC (com origem no Design/Ergonomia). 
Tal proximidade, metodológica, pode ser observada nos cartões lúdicos da IDEO (https://www.ideo.com/post/method-cards) e que podem ser utilizados como checklist para pesquisadores ou mesmo como elemento do projeto pedagógico a ser inserido no ensino na Graduação e na Pós-Graduação em Arquitetura e Urbanismo e em Design. Além disso, instrumentos como entrevistas, questionários, modelos em escala, mapas comportamentais e outros, subsidiam pesquisas qualitativas e quantitativas. Nesta linha se destacam, no Brasil, as obras publicadas por Pinheiro e Günther (2008), Pinheiro e Elali (2010), Villa e Ornstein (2013) e Ornstein (2016).

Essas interfaces, benéficas não só para as pesquisas, mas também para o ensino de APO (curso de Arquitetura e Urbanismo) e de Usabilidade de produtos (Curso de Design) puderam ser observadas nos últimos 10 anos na disciplina de graduação "Usabilidade e Desempenho" do Curso de Design da Faculdade de Arquitetura e Urbanismo da USP, ressaltando-se a facilidade com que os estudantes se utilizam dos instrumentos comuns para realizar as pesquisas que irão fundamentar intervenções e adequações de produtos (ORNSTEIN et al., 2011).

Portanto, na medida em que a APO requerer, para a sua implementação, ambientes qualificados (contemplando o mobiliário e sua infraestrutura, já em uso), a importância e a necessidade de investigar as interfaces APO - AEAC (aqui incorporando, os conhecimentos sobre Ergonomia), ficam bastante evidentes.

\section{APO, O NOVO E O QUE AINDA ESTÁ POR VIR}

De fato, nos últimos 20 anos a APO sofreu diversos aprofundamentos em suas abordagens metodológicas, o que vem significando avanços acadêmicos gradativos, mesmo que em alguns casos os resultados ainda sejam tímidos e careçam de maior desenvolvimento. Dentre estas evoluções podem ser citados:

- Listagem e testagem vigorosa de diversos métodos e técnicas (instrumentos) oriundos das ciências sociais, hoje disponibilizados a todos os pesquisadores interessados (PINHEIRO, GÜNTHER, 2008; RHEINGANTZ et al., 2009; VILLA, ORNSTEIN, 2013).

- Concepção e desenvolvimento de quadros sinópticos e mapas de descobertas ou mapas de diagnósticos e de recomendações, contemplando e resumindo os resultados da APO, o que amplia/facilita seu entendimento e seus possíveis rebatimentos nas atividades de projeto (RHEINGANTZ et al., 2009; VILLA, ORNSTEIN 2013).

- Incorporação da automação na coleta e no processamento de dados de campo da APO (FABRICIO, ONO, 2015; VILLA, SARAMAGO, GARCIA, 2016).

- Inserção das atividades de APO no processo de projeto, construção, uso, operação e manutenção de ambientes construídos, sobretudo por meio da tecnologia BIM (FRANÇA, 2016).

- Inserção de análise de custos versus benefícios para as recomendações oriundas da APO (ROMÉRO, ORNSTEIN, 2003).

- Aplicação da APO a estudos de casos de alta complexidade e envolvendo categorias bastante diversificadas de usuários, como hospitais e estações metroviárias, nos quais, foi possível realizar diagnósticos e recomendações relativas a fluxos, wayfinding, acessibilidade e segurança contra incêndio (ultrapassando, portanto, a fase dos relevantes, porém muito recorrentes, estudos de caso em habitação e escola)

- Desenvolvimento de instrumentos específicos de APO com base na NBR 15575 (ABNT, 2013), voltados para a avaliação de sistemas construtivos habitacionais denominados inovadores (FABRICIO, ONO, 2015).

- Valorização e incorporação das exigências éticas aos estudos de APO, com a autorização por Comitês de Ética em Pesquisa (Plataforma Brasil) para realização de levantamentos junto a usuários (realização de entrevistas, grupos focais, aplicação de questionários, etc.).

- Incorporação, de forma definitiva, da APO como parte da gestão da qualidade no processo de projeto, na medida em que ela se inseriu no Grupo de Trabalho (GT) Qualidade do Projeto da Associação Nacional de Tecnologia do Ambiente Construído (www.antac.org.br), passando a ser tratada não mais como um GT isolado ("GT-APO"), o que ampliou seus impactos na própria comunidade acadêmica em que se insere. 
Neste contexto, explorar mais as relações e possíveis resultados entre APO e a AEAC, além da intensificação dos estudos sobre os itens acima elencados, na forma de dissertações, teses e pesquisas nacionais em redes, deveria ser colocado nas agendas presente e futura das pesquisas nesse campo.

\section{O NOVO E A NOVIDADE: PRÓXIMOS PASSOS}

No item anterior foram destacados aspectos da Avaliação Pós-Ocupação cujo desenvolvimento está em curso e que poderão sofrer grande evolução nos próximos anos. É o caso, por exemplo, da automação na coleta e no processamento de dados e no caso do uso do BIM, em que as pesquisas acompanham a evolução de tantas outras; nos anos 1980, quando a APO teve início no Brasil, a computação era inexistente ou incipiente, e, portanto, pouco comparecia na maioria das pesquisas de então. Estes aspectos dizem respeito a incrementos metodológicos gradativos e estão muito associados a aplicabilidade da APO na prática profissional de arquitetos, urbanistas e engenheiros civis.

Mas seria possível, nos próximos anos, avanços ainda maiores, em termos teóricos e conceituais? Uma verdadeira revolução? Uma revolução seria necessária? O que há de novo (ou de novidade) nesta fronteira da APO?

Essas não são respostas fáceis e requerem, tal como nos incrementos metodológicos em curso, significativos esforços de disseminação do conhecimento (teórico-conceitual), discussão e amadurecimento de ideias e simultaneamente em diversos grupos de pesquisa de distintas universidades brasileiras em sintonias com outras estrangeiras.

Espera-se, finalmente, que os avanços conseguidos até o momento sejam suficientes não apenas para estimular os jovens pesquisadores a continuarem a estudar o tema, em todas as suas perspectivas, mas também para que esses avanços venham a significar o encurtamento dos prazos para as próximas etapas.

\section{REFERÊNCIAS}

ABRAHÃO, J.; SZNELWAR. L. I.; SILVINO, A.; SARMET, M.; PINHO, D. Introdução à Ergonomia: da prática à teoria. São Paulo: Editora Edgard Blücher, 2009. 240p.

ASSOCIAÇÃO BRASILEIRA DE NORMAS TÉCNICAS (ABNT). Edificações Habitacionais - Desempenho (NBR 15575) Partes 1 a 5. Associação Brasileira de Normas Técnicas. Rio de Janeiro. 2013.

IDEO. Methods Cards. Disponível em: https://www.ideo.com/post/method-cards. Acesso em: 29.07.2017.

FABRICIO, M. M.; ONO, R. (Orgs.). Avaliação de desempenho de tecnologias construtivas inovadoras. Manutenção e Percepção dos Usuários. Porto Alegre: ANTAC, 2015. 143p. Disponível em: file:///C:/Users/Sheila/AppData/Local/Temp/Avaliacao_de_desempenho_de_tecnologias_c-1.pdf. Acesso em: 30 de julho de 2017.

FRANÇA, A. J. G. L. F. Melhoria contínua aplicada à edificação de tipologia padronizada: a gestão do conhecimento no contexto do portfólio de ativos de uma organização. São Paulo: Faculdade de Arquitetura e Urbanismo da Universidade de São Paulo, 2016. Tese de Doutorado. Disponível em: http://www.teses.usp.br/teses/disponiveis/16/16132/tde07102016-114149/. Acesso em: 30 de julho de 2017.

LAWSON, B. Como arquitetos e designers pensam. São Paulo: Oficina de Textos, 2011. 296p.

MORAES, D. Metaprojeto. O Design do Design. São Paulo: Editora Edgard Blücher, 2010. 228p.

MONT'ALVÃO, C.; VILLAROUCO, V. (Orgs.). Um Novo Olhar para o Projeto. A Ergonomia no Ambiente Construído. Teresópolis, RJ: 2AB, 2011. 184p.

NORMAN, D. A. O Design do Dia a Dia. Rio de Janeiro: Rocco, 2006. 271p.

ORNSTEIN, S. W. Com os usuários em mente: um desafio para a boa prática arquitetônica? In: PARC Pesquisa em Arquitetura e Construção V.7., n.3. Campinas: UNICAMP, 2016. pp. 180-197. Disponível em: file:///C:/Users/Sheila/AppData/Local/Temp/8647437-25775-1-PB.pdf.

ORNSTEIN, S.; ROMÉRO, M. A.; FAGÁ, M. T. W.; SANTOS, E. M. (Orgs./Edits.). Design, Usabilidade \& Desempenho. Um exercício didático aplicado a objetos voltados à utilização do gás como fonte energética doméstica. São Paulo: Faculdade de Arquitetura e Urbanismo da Universidade de São Paulo, 2011. 97p.

ORNSTEIN, S. W. Desempenho do Ambiente Construído, Interdisciplinariedade e Arquitetura. São Paulo: Faculdade de Arquitetura e Urbanismo da Universidade de São Paulo, 1996. 53p. 
PINHEIRO, J. Q.; ELALI, G. A. (Orgs.). Inter-Ações Pessoa - Ambiente. Nove Estudos Potiguares. Natal, RN: Editora da Universidade do Rio Grande do Norte, 2010. 188p.

PINHEIRO, J. Q.; GüNTHER, H. (Orgs.). Métodos de Pesquisa nos Estudos Pessoa - Ambiente. São Paulo: Casa do Psicólogo, 2008. 396p.

RHEINGANTZ, P. A.; PEDRO, R. M. L. R.; SZAPIRO, A. M. (Orgs.). Qualidade do Lugar e Cultura Contemporânea. Modos de ser e habitar as cidades. Porto Alegre: Sulina, 2016. 398p.

RHEINGANTZ, P. A.; AZEVEDO, G. A. N.; BRASILEIRO, A.; ALCANTARA, D.; QUEIROZ, M. Observando a qualidade do lugar. Procedimentos para a avaliação pós-ocupação. Rio de Janeiro: PROARQ / UFRJ, 2009. 117p. Disponível em: http://www.fau.ufrj.br/prolugar/assets/obs_a_qua_lugar.pdf Acesso em: 30.07.2017.

ROMÉRO, M. A.; ORNSTEIN, S. W. Avaliação Pós-Ocupação. Métodos e Técnicas aplicados na Habitação Social. Porto Alegre: Associação Nacional de Tecnologia do Ambiente Construído, $2003.294 p$. www.habitare.org.br/publicacao_colecao1.aspx. Acesso em: 30 de julho de 2017.

ROSSI, P. H.; FREEMAN, H. E. Evaluation: a Systematic Approach. Newbury Park, California, EUA: Sage Publications, 1985. 423p.

VILLA, S. B.; ORNSTEIN, S. W. (Orgs.). Qualidade Ambiental na Habitação. São Paulo: Oficina de Textos, 2013. 400p.

VILLA, S. B.; SARAMAGO, R. C. P.; GARCIA, L. C. (2015). Avaliação Pós-Ocupação no Programa Minha Casa Minha Vida. Uma Experiência Metodológica. Uberlândia: Universidade Federal de Uberlândia. Disponível em https://morahabitacao.files.wordpress.com/2015/07/os-014631-proex-ufu-livro-sangria-lu.pdf Acesso em: 17 Nov., 2016.

ZEISEL, J. Inquiry by Design. Environment / Behavior / Neuroscience in Architecture, Interiors, Landscape, and Planning. New York: W.W.Norton \& Company, 2006. 400p.

NOTA DO EDITOR (*) O conteúdo do artigo e as imagens nele publicadas são de responsabilidade do(s) autor(es). 